\title{
Risk Stratification in Heart Failure Using ${ }^{123}$ I-MIBG
}

\author{
Geoffrey M. Currie ${ }^{1,2}$, Basit Iqbal ${ }^{1}$; Janelle M. Wheat ${ }^{1,2}$, Lexin Wang ${ }^{1,3}$, Marko Trifunovic ${ }^{4}$, Herbert F. Jelinek ${ }^{1}$, \\ and Hosen Kiat ${ }^{1,2,4,5}$ \\ ${ }^{I}$ Faculty of Science, Charles Sturt University, Wagga Wagga, Australia; ${ }^{2}$ Australian School of Advanced Medicine, Macquarie \\ University, Sydney, Australia; ${ }^{3}$ Department of Cardiology, Liaocheng Clinical School of Taishan Medical University, Taishan, China; \\ ${ }^{4}$ Macquarie Medical Imaging, Sydney, Australia; and ${ }^{5}$ Cardiac Health Institute, Sydney, Australia
}

\begin{abstract}
Heart failure is a progressive, heterogeneous form of cardiovascular disease that requires treatment to be individualized depending on the presenting symptoms. A decision to use an implantable cardioverter-defibrillator (ICD) is based on chronic heart failure patients presenting with a New York Heart Association classification of II or III and a left ventricular ejection fraction (LVEF) less than or equal to 30\%-35\%. A large percentage of ICD devices, however, never deliver therapy during their lifetime, and as many as 33\% of patients ineligible for an ICD (LVEF $>35 \%$ ) die of sudden cardiac death. ${ }^{123}$ I-metaiodobenzylguanidine (123/-MIBG) scintigraphy identifies sympathetic nervous system dysfunction and has been shown to lead to better patient stratification. This article reviews the role of planar 123/-MIBG global quantitation in improving differentiation of heart failure, regardless of the LVEF, to better identify those in whom an ICD is more likely to reap benefits. It goes on to explore the potential incremental benefit of SPECT-based regional quantitation to risk stratification and provides a case example in which ${ }^{123}$ I-MIBG SPECT was used to inform a decision to not use an ICD in a patient eligible under the standard criteria.
\end{abstract}

Key Words: SPECT; heart failure; MIBG

J Nucl Med Technol 2011; 39:295-301

DOI: 10.2967/jnmt.111.088369

\section{H} eart failure is a progressive, heterogeneous form of cardiovascular disease that requires treatment to be individualized depending on the presenting symptoms. Heart failure is the inability of the left ventricle to fill or eject enough blood to meet the requirements of the body (1) and might be viewed as a syndrome representative of a variety of cardiac diseases (2). Nonetheless, coronary artery disease is the underlying pathology in $70 \%$ of heart failure patients (3). Chronic heart failure involves both mechanical failure

\footnotetext{
Received Jan. 21, 2011; revision accepted Jul. 5, 2011.

For correspondence or reprints contact: Geoffrey M. Currie, Faculty of Science, Locked Bag 588, Charles Sturt University, Wagga Wagga 2678, Australia.

E-mail: gcurrie@csu.edu.au

Published online Oct. 3, 2011

COPYRIGHT @ 2011 by the Society of Nuclear Medicine, Inc.
}

and autonomic nervous system dysfunction that can lead to sudden cardiac death (SCD). Several therapies are therefore utilized, including pharmaceutical and device options-for example, the implantable cardioverter-defibrillator (ICD) device. Despite these options, the long-term prognosis in heart failure is poor, with 5-y mortality rates of $59 \%$ and $45 \%$ for men and women, respectively (3). There is a clear need for improved stratification of patients based on risk, yet there is a paucity of screening programs available for early detection (2).

A decision to use an ICD is based on chronic heart failure patients presenting with a New York Heart Association (NYHA) classification of II or III and a left ventricular ejection fraction (LVEF) less than or equal to $30 \%-35 \%$, depending on the country. A large percentage of ICD devices, however, never deliver therapy during their lifetime, and as many as $33 \%$ of patients ineligible for an ICD (LVEF > 35\%) die of SCD (4). Consequently, there is a pressing need for improved differentiation of chronic heart failure, regardless of the LVEF, to better identify those in whom an ICD is more likely to reap benefits. In the United States, scintigraphy with ${ }^{123}$ I-metaiodobenzylguanidine ( $\left.{ }^{123} \mathrm{I}-\mathrm{MIBG}\right)$ has been used for risk stratification in chronic heart failure patients with low LVEF $(<35 \%)$. ${ }^{123}$ I-MIBG identifies sympathetic nervous system dysfunction and has been shown to lead to better patient stratification (4).

\section{HEART FAILURE}

Heart failure has been described as the "Cinderella of health issues-hardly registering on the radar of key health care providers, regulators, relevant government bodies and the general public" (5). This is in a large part due to a lack of a universally recognized definition of heart failure and because varying degrees of left ventricular systolic dysfunction may be asymptomatic. Indeed, almost half of heart failure patients have preserved left ventricular function (1). In the United States, there are more than 5 million people with heart failure (1-3), and there are estimated to be more than 20 million heart failure patients worldwide (1). In the United States, 550,000 new heart failure cases 
are detected each year, more than 1 million heart failurerelated hospitalizations occur each year, and heart failure currently costs $\$ 29$ billion annually (3). For developed countries, $1 \%-2 \%$ of the population has heart failure $(1,6,7)$. Clark et al. (8) estimated that 325,000 Australians had symptomatic heart failure and another 214,000 had left ventricular systolic dysfunction, in a population of just 20 million. The same investigators estimated that heart failure in Australia was responsible for 1.4 million hospital days and $\$ 1$ billion in costs annually.

Age is a good predictor of the incidence of heart failure. This incidence is 10 per 1,000 people over $65 \mathrm{y}$ old, and $20 \%$ of hospitalizations are in those over 65 y old (2). For those 50-59 y old, the prevalence is 8 per 1,000 people for both men and women; however, this prevalence sharply increases for those 80-89 y old, to 66 per 1,000 and 79 per 1,000 for men and women, respectively (1).

Heart failure has a median survival of just $1.7 \mathrm{y}$ and $3.2 \mathrm{y}$ for men and women, respectively (1). It has a $10 \%$ mortality by $30 \mathrm{~d}$, which highlights an early high-risk period of disease. This high-risk period is followed by a 5-y mortality rate of $54 \%$ and $40 \%$ for men and women, respectively (9). Chronic heart failure is progressive and ultimately fatal, making early detection crucial for delaying progression. For patients with mild symptoms, the annual death rate is $5 \%-10 \%$, and this increases sharply to $30 \%-40 \%$ for those with more advanced symptoms (10).

Although heart failure is generally classified according to the NYHA classification (Table 1), there are other classification methods. The American College of Cardiology (ACC) and American Heart Association (AHA) offer an alternative classification (Table 1) that has several advantages over the NHYA version. The NYHA classification is based on functional limitations of the heart failure patient, progressing from lesser to greater limitation. Unlike the disease itself, the NYHA classification may regress if a patient's functional status improves. Conversely, the ACC/
AHA classification progresses from stage A to D and cannot reverse the order (2). This is an important discussion because the decision to provide or refuse an ICD in heart failure based on the NYHA classification of the patient may not reflect the patient's actual disease stage. That is, functional status may improve from NYHA IV to III without an improvement in disease state or, indeed, risk. Stage D in the ACC/AHA classification, on the other hand, provides a consistent classification of disease state and risk.

Morbidity and mortality in chronic heart failure remain high, despite advances in treatment (8). There are several approaches to management of chronic heart failure, and these generally relate to the severity of disease, symptoms, and comorbidity. The goal of therapy is to improve and prolong life, and this is achieved using lifestyle modifications, pharmaceutical therapy, surgery, supportive devices, management programs, and palliative care (6). More recently, cardiac resynchronization therapy has emerged as a good option for those patients presenting with class III or IV heart failure (11). The risk of arrhythmia and SCD is greater in patients with CHD. Consequently, ICD devices have been used in select patients for over a decade to protect against arrhythmia and SCD and have been increasingly used in Australia; 2,864 were implanted in 2005, a number that was up from 956 in 2001 (12). The ICD is a device that combines the functions of a traditional pacemaker, a Holter monitor, and a defibrillator. The Cardiac Society of Australia and New Zealand has stated that ICDs are indicated in chronic heart failure patients with an LVEF less than $35 \%$ and NYHA class II or III symptoms (6). Medicare Australia provides coverage for chronic heart failure patients who have NYHA class II or III symptoms or an LVEF of less than or equal to $35 \%$ and have received optimized medical therapy. Patients treated with an ICD have a $1 \%-3 \%$ annual decrease in absolute mortality, which equates to a $20 \%-30 \%$ relative reduction in mortality each year. VALIANT (Valsartan in Acute Myocardial Infarction

TABLE 1

NYHA and ACC/AHA Classifications for Heart Failure

\begin{tabular}{|c|c|c|c|}
\hline \multicolumn{2}{|l|}{ NYHA classification } & \multicolumn{2}{|r|}{ ACC/AHA classification } \\
\hline Description & Class & Class & Description \\
\hline & - & A & $\begin{array}{l}\text { High risk of developing heart failure but no functional } \\
\text { or structural heart deficits }\end{array}$ \\
\hline No limitation and no symptoms from ordinary activity & I & B & Structural heart deficit but no symptoms \\
\hline $\begin{array}{l}\text { Mild limitation with activity and comfortable at } \\
\text { rest or with mild exertion }\end{array}$ & II & $\mathrm{C}$ & $\begin{array}{l}\text { Heart failure symptoms due to underlying structural } \\
\text { heart deficit with medical management }\end{array}$ \\
\hline $\begin{array}{l}\text { Significant limitation with any activity and comfortable } \\
\text { only at rest }\end{array}$ & III & C & $\begin{array}{l}\text { Heart failure symptoms due to underlying structural } \\
\text { heart deficit with medical management }\end{array}$ \\
\hline $\begin{array}{l}\text { Discomfort with any physical activity and symptoms } \\
\text { occurring at rest }\end{array}$ & IV & $\mathrm{D}$ & $\begin{array}{l}\text { Advanced disease requiring hospitalization, transplant, } \\
\text { or palliative care }\end{array}$ \\
\hline
\end{tabular}

Arrows indicate potential directions of stage progression. Horizontal alignment indicates corresponding stages between the 2 classifications. 
Trial) reported on 14,609 patients with left ventricular systolic dysfunction or heart failure after myocardial infarction and found a mortality rate of $6.2 \%$ after a median $24.7-\mathrm{mo}$ follow-up (13). Analysis revealed that each decrease of 5 points in LVEF was associated with a $21 \%$ adjusted increase in risk of mortality (13). MADIT-CRT (Multicenter Automatic Defibrillator Implantation Trial with Cardiac Resynchronization Therapy) recruited 1,232 patients with previous myocardial infarction and an LVEF less than $30 \%$ and reported that, compared with standard medical therapy, the addition of an ICD improved the 20-mo follow-up mortality rate from $19.8 \%$ to $14.2 \%$ (14).

\section{CARDIAC AUTONOMIC NEUROPATHY IN HEART FAILURE}

The compensatory response of the autonomic nervous system to left ventricular dysfunction in heart failure becomes part of the disease progress leading eventually to further deterioration. The sympathetic nervous system is part of this response. The ventricular innervation is characterized by a gradient from base to apex (15). Chronic impairment in cardiac output due to damage to the cardiac pump increases plasma noradrenaline levels released from sympathetic postganglionic nerves. This sympathetic overdrive, which is related to dysfunctional atrial baroreceptor reflex activity and sympathovagal balance, parallels the clinical severity of heart failure expressed by the NYHA functional class categories and is similar in ischemic or nonischemic heart failure states (16).

\section{3|-MIBG IN HEART FAILURE}

Molecular imaging of the cardiac sympathetic neuronal system is possible with ${ }^{123}$ I-MIBG because it is a false adrenergic neurotransmitter that has a reuptake mechanism similar to that of norepinephrine. Fortunately, ${ }^{123}$ I-MIBG is not metabolized and does not interact with postsynaptic receptors, thus allowing scintigraphic imaging at early (15 min after administration) and delayed (4 $\mathrm{h}$ after administration) phases (17). Impaired sympathetic innervation has been shown to increase ventricular arrhythmias because even in viable myocardium the impaired innervations result in abnormal chemical and electrical stimulation (18). Because the sympathetic nervous tissue is more sensitive to ischemia than the myocardium, impaired innervations can cause arrhythmia but be insufficient to cause myocardial infarction (18). Clinical use is well established for ${ }^{123}$ I-MIBG, and its safety profile is well documented to provide a useful noninvasive tool to risk-stratify patients with chronic heart failure. Greater deprivation of cardiac sympathetic innervation is associated with a worse prognosis in heart failure patients (19).

Generally speaking, the delayed uptake (heart-to-mediastinum ratio $[\mathrm{H}: \mathrm{M}]$ ) has been shown to have predictive power although washout rate and defect score have also been reported to be clinically important. Nonetheless, the correlation between LVEF and H:M is weak $(r=0.50)$ (20). Although chronic heart failure can show an initial compensatory increase in myocardial uptake of ${ }^{123}$ I-MIBG, low uptake is strongly correlated with a poor prognosis and predicts an increased risk of arrhythmia and SCD (21). More recently, the washout rate of ${ }^{123}$ I-MIBG from the myocardium between early and delayed images has shown promising predictive power (3). Heart failure is associated with more rapid washout, and even in the presence of heart failure, the washout rates of lung, liver, and mediastinum appear uniform (21). Washout greater than $27 \%$ has been reported as a strong predictor of survival but not necessarily of cardiac events (22). One might consider the late uptake a marker for risk of SCD and washout a marker of survival independent of SCD risk (i.e., death due to disease progression). Together, they might provide better stratification of those suitable for ICD, with uptake identifying those likely to benefit and washout those less likely to benefit. Table 2 provides an overview of the key heart failure studies evaluating ${ }^{123}$ I-MIBG.

There has been a broad range of research and reports in the medical literature highlighting the role ${ }^{123}$ I-MIBG plays in risk stratification. Recently, a prospective multinational, multicenter open-label, phase 3 study (ADMIRE [AdreView Myocardial Imaging for Risk Evaluation in Heart Failure]) evaluated the role of ${ }^{123}$ I-MIBG imaging in identifying chronic heart failure patients who are likely to expe-

TABLE 2

Global ${ }^{123}$ I-MIBG Quantitative Values from Various Studies

\begin{tabular}{clccc}
\hline Investigator & Parameter & Healthy cohort & Heart failure cohort & $P$ \\
\hline Matsuo et al. (25) & LVEF (\%) & $73 \pm 7$ & $31 \pm 1$ & $1.8 \pm 0.9$ \\
& Delayed H:M & $2.6 \pm 0.3$ & $38 \pm 3$ & $<0.05$ \\
& Washout (\%) & $28 \pm 3$ & $22.0 \pm 8.4$ & $<.05$ \\
Zhao et al. (26) & LVEF (\%) & $71.0 \pm 11.2$ & $1.73 \pm 0.39$ & - \\
& Delayed H:M & $2.66 \pm 0.21$ & $31.58 \pm 11.18$ & - \\
& Washout (\%) & $11.88 \pm 7.23$ & $2.36 \pm 0.66$ & 0.04 \\
Bengel et al. (27) & Delayed H:M & $2.8 \pm 0.55$ & $11.6 \pm 7.9$ & 0.002
\end{tabular}

Data are mean $\pm S D$. 
rience a major adverse cardiac event (23). The study included 961 patients with a NYHA II or III classification and an LVEF of $35 \%$ or less who were followed for $2 \mathrm{y}$. The results indicated that normal (using a delayed H:M of 1.60 as the cutoff) ${ }^{123}$ I-MIBG uptake was associated with an $85 \%$ event-free $2-y$ period whereas abnormal ${ }^{123}$ I-MIBG had only a $63 \%$ 2-y event-free period. The investigation also showed that H:Ms less than 1.20 had a 10 -fold increase in event rate over those greater than or equal to 1.60. Carrio et al. (9) used a delayed H:M of 1.75 as the cutoff and improved sensitivity to $84 \%$ (specificity, 60\%) for prediction of events, with a $95 \%$ event-free survival for $2 \mathrm{y}$ greater than or equal to 1.75 and $62 \%$ for those lower than $1.75(9)$.

In a 2-y follow-up in chronic heart failure patients, $21 \%$ of patients underwent ${ }^{123}$ I-MIBG SPECT to document that ICD discharge was appropriate, and patients with greater defect scores showed a 10-fold higher likelihood of appropriate ICD discharge therapy (24). In 116 patients who were referred for ICD and presented with advanced heart failure, Boogers et al. (24) showed that a high defect score $(<26)$ determined by regional summation on SPECT was predictive of appropriate ICD discharge. The late ${ }^{123}$ I-MIBG defect score was also independently predictive of appropriate ICD discharge. Indeed, patients with a late ${ }^{123} \mathrm{I}-\mathrm{MIBG}$ SPECT summed defect score greater than 26 had a $52 \%$ appropriate discharge rate, compared with just $5 \%$ for those with lower defect scores $(P<0.01)$.

\section{CLINICAL CASE}

A 77-y-old man presented with dyspnea on exertion and ischemic cardiomyopathy secondary to a history of myocardial infarction in the inferior wall and coronary artery bypass grafting performed $4 \mathrm{y}$ earlier, a saphenous vein graft to the first and second obtuse marginal arteries, a saphenous vein graft to the posterior descending artery, and a pedicled left internal mammary artery to the left anterior descending artery. The resting electrocardiogram demonstrated atrial fibrillation, left anterior hemiblock, and poor R-wave progression. Postsurgical atrial fibrillation had been treated with sotalol. No significant ST deviation was noted on the stress electrocardiogram. Stress myocardial perfusion imaging was performed as dipyridamole pharmacologic stress in conjunction with hand-grip exercise. Resting myocardial perfusion SPECT evaluation revealed a left ventricular end-diastolic volume of $195 \mathrm{~mL}$ at rest and $210 \mathrm{~mL}$ after stress. The LVEF was $32 \%$ at rest and $34 \%$ after stress. The myocardial perfusion SPECT study revealed a fixed perfusion defect in the inferior and inferolateral walls. A partially reversible perfusion defect was noted in the anterolateral wall. Wall motion was paradoxic in the septal wall, akinetic in the inferior wall, and severely hypokinetic in the inferolateral wall. On the basis of this history, the patient was eligible for ICD implantation under Australian Medicare coverage.

The patient was administered $185 \mathrm{MBq}$ of ${ }^{123} \mathrm{I}-\mathrm{MIBG}$ intravenously and received $30 \mathrm{mg}$ of Lugol iodine daily from $2 \mathrm{~d}$ before ${ }^{123}$ I-MIBG administration until $2 \mathrm{~d}$ after ${ }^{123}$ I-MIBG administration. The early-phase imaging commenced 15 min after intravenous administration using 600s anterior planar chest images. A 256 matrix on an Infinia Hawkeye 4 scanner (GE Healthcare) with medium-energy general-purpose collimation was used. Planar imaging was followed by $180^{\circ}$ SPECT imaging $\left(45^{\circ}\right.$ left posterior oblique to $45^{\circ}$ right anterior oblique) using a 64 matrix and 60 projections at $25 \mathrm{~s}$ per projection. The patient was imaged supine with the arms extended above the head. The SPECT study was reconstructed using CT-based attenuation correction. The planar and SPECT acquisitions were repeated $4 \mathrm{~h}$ after intravenous administration. Global myocardial, mediastinal, lung, and liver regions of interest were applied to the planar images. Normalized (counts per pixel) H:Ms were calculated for both the early phase (2.15) and the delayed phase (2.21) using previously described methods (25-27). Delayed image statistics were decayed-corrected. The global washout rate from early to delayed phases was calculated for myocardium, mediastinum, lung, and liver. The cardiac washout rate was determined as $35.0 \%$ by the following equation (25-27):

\section{[early-phase counts per pixel - delayed-phase counts per pixel $] \times 100$ early-phase counts per pixel}

Regions were applied to the planar image as described by Verberne et al. (28), with the myocardium represented by a region that circumscribes the entire myocardium and the mediastinum region placed adjacent to the upper part of the lung on the midline of the chest (Fig. 1). High lung uptake of ${ }^{123}$ I-MIBG is characteristic of heart failure and represents a limitation of region-of-interest placement (21). Given the intense lung uptake in this patient and the potential that lung counts might contribute to the myocardial counts, a smaller region was also drawn to include representative myocardial activity with exclusion of lung superimposition (21). A duplicate mediastinal region was also drawn adjacent to the lower third of the lung. H:Ms and washout rates were determined using the various combinations to assess how robust the calculations were (Table 3). Regions of interest were also placed on the liver and each lung. Verberne et al. (21) reported washout rates to be elevated in the myocardium and decreased in the liver in chronic heart failure patients, compared with healthy patients and patients with hypertension. In this patient, liver washout was $0 \%$, and the right and left lungs showed $46.7 \%$ and $48.4 \%$ washout, respectively. Although the global H:M, despite the poor LVEF, demonstrated a low risk of SCD (ratio well in excess of 1.75), the global washout rate was abnormal. The H:M reflects cardiac sympathetic activity, whereas the washout rate reflects norepinephrine retention by sympathetic neurons. Thus, more rapid washout might indicate the need to decide against an ICD. 


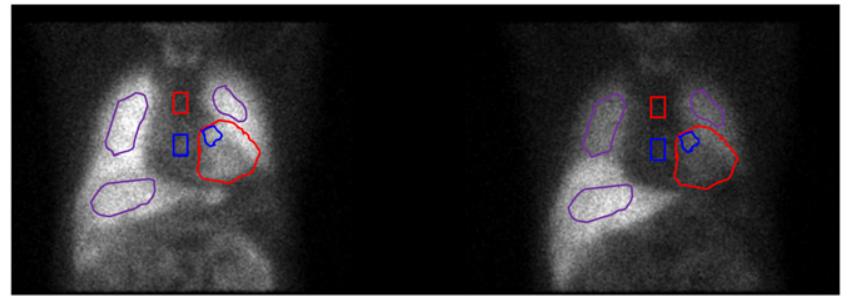

FIGURE 1. Anterior planar images demonstrating global accumulation of ${ }^{123}$ I-MIBG in myocardium at $15 \mathrm{~min}(\mathrm{~A})$ and 4 $h(B)$ after injection. Standard global regions of interest are red; modified regions of interest are blue. Lung and liver regions are purple. Images highlight need for greater discriminatory power using SPECT. Nonetheless, H:M ratio is on order of 2 , and washout is marked at $4 \mathrm{~h}$.

Normalized (counts per pixel) regions of interest were drawn on mid short-axis and vertical long-axis SPECT slices (26). On the short-axis slice, regions included anterior, septal, lateral, and inferior walls. On the vertical longaxis slice, the region included the apex. Matsuo et al. (29) used $5 \times 5$ pixel regions in the basal and distal short-axis slice for anterior, lateral, septal, and inferior walls, plus the apex on the vertical long-axis slice. Our approach was to use the mid short-axis slice and mid vertical long-axis slice to generate, for each wall, larger regions that represented the entire territory (i.e., anterior, lateral, septal, inferior, and apical). The 4-h data were decay-corrected, and regional washout rates and uptake index were determined (Table 4) using the method described by Zhao et al. (26) (Table 5):

\section{Regional washout rate}

$=[$ mean early segment counts - mean delayed segment counts $] \times 100$ mean early segment counts

$$
\text { Uptake index }=\frac{\text { mean segment counts } \times \mathrm{H}: \mathrm{M}}{\text { maximum voxel counts in all myocardial regions }} .
$$

Polar maps were generated to demonstrate regional cardiac ${ }^{123}$ I-MIBG distribution at $15 \mathrm{~min}$ (left) and $4 \mathrm{~h}$ (right) after intravenous administration (Fig. 2). The polar maps show the marked washout from the apex and moderate washout from the inferolateral wall. The cascade of heart failure sympathetic dysfunction can be appreciated in the inferior wall, progressing into the lateral and septal walls and sparing the anterior wall at this point in the disease. The areas of denervation correspond to similar regions of hypoperfusion on the end-diastolic polar map in the 99mTc-MIBI study (Fig. 3) but show thickening and motion (Fig. 4). Figures 2 and 3 provide a comparison of the innervation and perfusion to the myocardium. The inferior septum, with its paradoxic motion, has a more extensive ${ }^{123}$ I-MIBG defect than the perfusion. Similarly, the inferior lateral wall and the apex show discordance between ${ }^{123} \mathrm{I}-$ MIBG and ${ }^{99 m}$ Tc-MIBI perfusion. Indeed, both wall

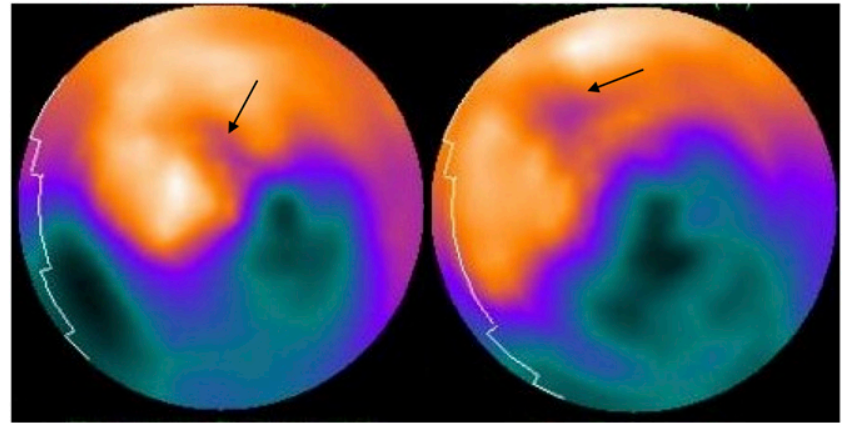

FIGURE 2. Polar map demonstrating regional cardiac ${ }^{123}$ |-MIBG distribution at $15 \mathrm{~min}$ (left) and $4 \mathrm{~h}$ (right) after intravenous administration. Arrows highlight areas of denervation that correspond to similar regions of hypoperfusion on end-diastolic polar map in ${ }^{99 m}$ Tc-MIBI study (Fig. 3) but show thickening and motion (Fig. 4).

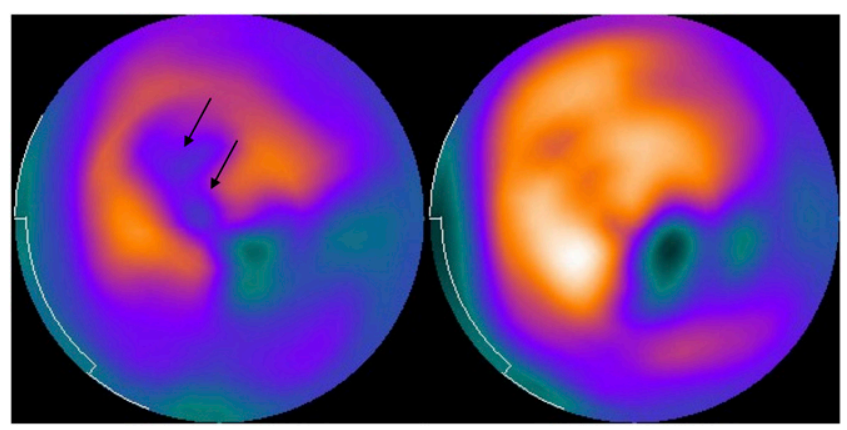

FIGURE 3. Polar map demonstrating regional myocardial perfusion (\%) at end-diastole (A) and end-systole (B). Perfusion deficits are seen in inferior and inferolateral walls. Arrows highlight areas of decreased perfusion in distal left anterior descending region that correspond to similar regions of ${ }^{123}$ |-MIBG denervation (Fig. 3).

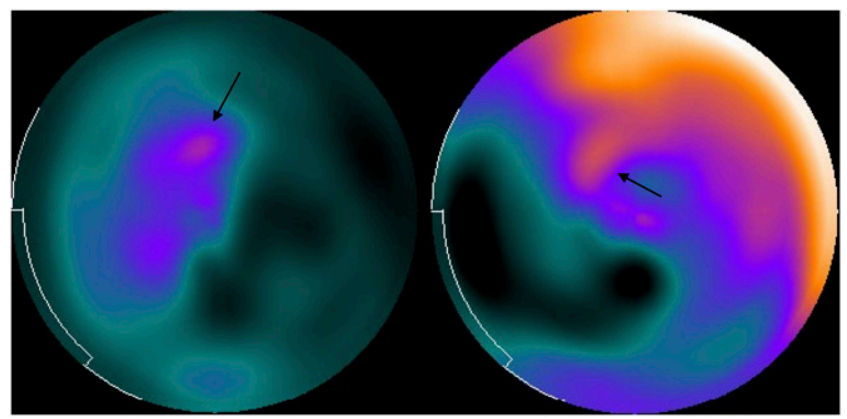

FIGURE 4. Polar map demonstrating regional myocardial thickening (A) and wall motion (B). Arrows highlight areas of decreased perfusion and denervation in distal left anterior descending region that show both thickening and motion. Septal paradoxic motion can be appreciated, and inferior and inferolateral hypokinesia is also apparent.

motion and wall thickening are noted in these regions on the perfusion study. The global ${ }^{123}$ I-MIBG findings reflect sympathic change associated with infarction, not just progression of heart failure. Interestingly, the non-myocardial 
TABLE 3

$\mathrm{H}: \mathrm{M}$ and Washout Rates for Optimistic and Pessimistic Approaches to Region-of-Interest Placement

\begin{tabular}{lcccc}
\hline Parameter & $\begin{array}{c}\text { Whole heart/upper } \\
\text { mediastinum }\end{array}$ & $\begin{array}{c}\text { Whole heart/lower } \\
\text { mediastinum }\end{array}$ & $\begin{array}{c}\text { Regional heart/upper } \\
\text { mediastinum }\end{array}$ & $\begin{array}{c}\text { Regional heart/lower } \\
\text { mediastinum }\end{array}$ \\
\hline Early H:M & 2.15 & 1.66 & 2.65 & 2.05 \\
Delayed H:M & 2.21 & 1.65 & 2.55 & 1.90 \\
Heart & $34.2 \%$ & $34.2 \%$ & $38.3 \%$ & $38.3 \%$ \\
Washout & & & &
\end{tabular}

Tabulated data show some variability; however, best and worst cases are consistent with low-risk H:M and abnormal washout.

infarction region of greatest ${ }^{123}$ I-MIBG washout (apex) was also the region with the greatest thickening.

Despite satisfying the criteria to receive an ICD under the Medicare scheme, an ICD was not implanted and the patient remained event-free at 12 mo of follow-up.

\section{DISCUSSION}

The cost-effectiveness of ICD therapy remains controversial. Although ICDs have proven value in reducing mortality in chronic heart failure, they remain expensive. Therefore, identifying those patients who would benefit most from an ICD is crucial. SCD occurs more commonly in patients with an LVEF over $35 \%$, with $67 \%$ of SCD patients having an LVEF over 35\% and only $33 \%$ having an LVEF less than or equal to $35 \%$ (4). This no doubt reflects the high number of patients with an LVEF under $35 \%$ who die of progressive heart failure rather than SCD. In addition, a substantial number of patients with an ICD and LVEF less than $35 \%$ never see it deliver therapy during its lifetime. The ability to accurately stratify those patients with a low LVEF in whom an ICD is not indicated (discharge unlikely or chronic heart failure progression fatal) and those with a higher LVEF in whom it is (SCD likely) would minimize unnecessary health expenditure and decrease preventable deaths, respectively. There is a paucity of literature examining the predictive capability of ${ }^{123} \mathrm{I}-$ MIBG in the above-35\% LVEF group.

Reliance on LVEF is a poor predictor of who does and does not need an ICD. An LVEF greater than or equal to
$30 \%$ with a delayed ${ }^{123} \mathrm{I}-\mathrm{MIBG} \mathrm{H}: \mathrm{M}$ ratio greater than or equal to 1.60 is associated with zero deaths in the 2-y follow-up period (23). The same LVEF stratification, however, with an H:M ratio less than 1.60 has a greater cumulative 2-y mortality than those with an LVEF less than $30 \%$ and an H:M ratio greater than 1.60. Naturally, LVEF and an H:M ratio lower than $30 \%$ and 1.60 , respectively, are associated with the greatest $2-y$ mortality. There is no specific line above or below which a decision can be made to use ICD. Although the literature to date indicates ${ }^{123} \mathrm{I}-\mathrm{MIBG}$ to be a reliable and strong predictor of events, it does not provide sufficient discriminatory power to direct a decision to either offer ICD in those with a LVEF over $35 \%$ or to decline the ICD option in those with an LVEF below $35 \%$. The discriminatory power is perhaps reduced by the global approach to calculations. That is, cardiac autonomic neuropathy begins in the inferior wall of the heart and progresses through adjacent myocardial walls (lateral and septal) before eventually reaching the anterior wall (30). Normal global uptake may reflect anterior wall uptake and fail to highlight early cardiac autonomic neuropathy in the inferior wall.

There is a need for further evaluation of the role of both planar and SPECT ${ }^{123}$ I-MIBG imaging in stratification of heart failure patients-in particular, to more accurately identify patients with an LVEF less than $35 \%$ who are unlikely to benefit from the ICD, and to identify those with an LVEF over $35 \%$ in whom ICD is likely to be life-saving. This approach will result in more cost-effective heart fail-

TABLE 4

Regional Analysis on SPECT

\begin{tabular}{lccc}
\hline \multicolumn{1}{c}{ Parameter } & Uptake index & Regional washout rate (\%) & Washout index (uptake index) \\
\hline Early anterior & 1.67 & $40.9 \%$ & 0.93 \\
Delayed anterior & 1.79 & $33.9 \%$ & 0.83 \\
Early lateral & 0.85 & & \\
Delayed lateral & 1.02 & $56.9 \%$ & 1.29 \\
Early inferior & 0.58 & $34.1 \%$ & 0.84 \\
Delayed inferior & 0.45 & & \\
Early septal & 1.47 & $46.2 \%$ & 1.02 \\
Delayed septal & 1.76 & & \\
Early apical & 1.02 & & \\
Delayed apical & 1.00 & & \\
\hline
\end{tabular}


TABLE 5

Regional ${ }^{123}$-MIBG Parameters Determined by Zhao et al. (26) Using SPECT

\begin{tabular}{lcc}
\hline \multicolumn{1}{c}{ Parameter } & Healthy cohort & Heart failure cohort \\
\hline LVEF (\%) & $54.6 \pm 17.7$ & $16.8 \pm 9.6$ \\
Delayed MUP & $2.12 \pm 0.36$ & $1.23 \pm 0.52$ \\
Washout (\%) & $1.04 \pm 16.8$ & $38.18 \pm 18.52$ \\
& \\
\hline & \\
MUP $=$ uptake index. & \\
Data are mean \pm SD.
\end{tabular}

ure management, both decreasing health care costs and increasing lives saved. This case highlights a patient eligible for ICD based on standard criteria but in whom the global and regional ${ }^{123}$ I-MIBG study suggest that ICD implantation is not ideal.

\section{CONCLUSION}

Although ${ }^{123}$ I-MIBG imaging and global analysis provide a useful tool to direct appropriate heart failure treatment options in a patient eligible for ICD implantation, the discriminatory power of regional quantitation using SPECT data adds an important dimension that warrants further clinical investigation. Although delayed H:Ms provide improved stratification of heart failure patients over LVEF and heart failure classification, the washout rate provides an important marker for identifying more rapid disease progression and lower likelihood of a benefit from ICD. Further research is required for both global and regional quantitation in this patient group.

\section{ACKNOWLEDGMENT}

No potential conflict of interest relevant to this article was reported.

\section{REFERENCES}

1. Tendera M. The epidemiology of heart failure. J Rennin-Angiotensin-Aldosterone System. 2004;5(suppl):S2-S6.

2. Jessup M, Bronzena S. Heart failure. N Engl J Med. 2003;348:2007-2019.

3. Bax JJ, Boogers M, Henneman MM. Can cardiac iodine-123 metaiodobenzylguanidine imaging contribute to risk stratification in heart failure patients? Eur J Nucl Med Mol Imaging. 2008;35:532-534.

4. Gerson MC, Abdallah M, Muth JN, Costea AI. Will imaging assist in the selection of patients with heart failure for ICD? CC Cardiovasc Imaging. 2010;3:101-110.

5. Krum H, Stewart S. Chronic heart failure: time to recognise this major public health problem. Med J Aust. 2006;184:147-148.

6. National Heart Foundation of Australia and the Cardiac Society of Australia and New Zealand (Chronic Heart Failure Guidelines Expert Writing Panel). Guidelines for the Prevention, Detection and Management of Chronic Heart Failure in Australia. Sydney, Australia: National Heart Foundation of Australia; 2006.

7. Krum H, Jelinek MV, Simon S, Sindone A, Atherton JJ, Hawkes AL. Guidelines for the prevention, detection and management of people with chronic heart failure in Australia 2006. Med J Aust. 2006;185:549-556.
8. Clark RA, McKenna S, Dawson A, Wilkinson D, Stewart S. Uncovering a hidden epidemic: a study of the current burden of heart failure in Australia. Heart Lung Circ. 2004;13:266-273.

9. Carrió I, Cowie MR, Yamazaki J, Udelson J, Camici PG. Cardiac sympathetic imaging with mIBG in heart failure. JACC Cardiovasc Imaging. 2010;3:92-100.

10. Gomberg-Maitland M, Baran DA, Fuster V. Treatment of congestive heart failure: guidelines for the primary care physician and the heart failure specialist. Arch Intern Med. 2001;161:342-352.

11. Steffel J, Holzmeister J, Abraham WT. Recent advances in cardiac resynchronization therapy. Postgrad Med. 2011;123:18-26.

12. Mond HG, Whitlock RML. The Australian and New Zealand cardiac pacing and implantable cardioverter-defibrillator survey: calendar year 2005. Heart Lung Circ. 2008;17:85-89.

13. Soloman SD, Zelenkofske S, McMurray JJ, et al. Sudden death in patients with myocardial infarction and left ventricular dysfunction, heart failure, or both. $N$ Engl J Med. 2005;352:2581-2588.

14. Moss AJ, Hall WJ, Cannom DS, et al. Cardiac-resynchronization therapy for the prevention of heart-failure events. N Engl J Med. 2009;361:1329-1338.

15. Triposkiadis F, Karayannis G, Giamouzis G, Skoularigis J, Louridas G, Butler J. The sympathetic nervous system in heart failure. J Am Coll Cardiol. 2009;54: 1747-1762.

16. Grassi G, Seravalle G, Bertinieri G, et al. Sympathetic and reflex abnormalities in heart failure secondary to ischaemic or idiopathic dilated cardiomyopathy. Clin Sci. 2001;101:141-146.

17. Dilsizian V, Chandrashekhar Y, Narula J. Introduction of new tests: low are the mountains, high the expectations. J Am Coll Cardiol Imaging. 2010;3:117-119.

18. Simula S, Lakka T, Laitinen T, et al. Cardiac adrenergic denervation in patients with non-Q-wave versus Q-wave myocardial infarction. Eur J Nucl Med. 2000;27:816-821.

19. Boogers MJ, Fukushima K, Bengel FM, Bax JJ. The role of nuclear imaging in the failing heart: myocardial blood flow, sympathetic innervation, and future applications. Heart Fail Rev. 2011;16:411-423.

20. Merlet P, Valette H, Dubois-Rande JL, et al. Prognostic value of cardiac metaiodobenzylguanidine imaging in patients with heart failure. $J$ Nucl Med. 1992;33:471-477.

21. Verberne HJ, Somsen GA, Povinec P, van Eck-Smit BLF, Jacobson AF. Impact of mediastinal, liver and lung ${ }^{123} \mathrm{I}$-metaiodobenzylguanidine ( $\left.{ }^{123} \mathrm{I}-\mathrm{MIBG}\right)$ washout on calculated ${ }^{123}$ I-MIBG myocardial washout. Eur J Nucl Med Mol Imaging. 2009;36:1322-1328.

22. Yamada T, Shimonagata T, Fukunami M, et al. Comparison of the prognostic value of cardiac iodine-123 metaiodobenzylguanidine imaging and heart rate variability in patients with chronic heart failure: a prospective study. $J$ Am Coll Cardiol. 2003;41:231-238.

23. Jacobson AF, Senior R, Cerqueira MD, et al. Myocardial iodine-123 metaiodobenzylguanidine imaging and cardiac events in heart failure: results of the prospective ADMIRE-HF study. J Am Coll Cardiol. 2010;55:2212-2221.

24. Boogers MJ, Borleffs CJW, Henneman MM, et al. Cardiac sympathetic denervation assessed with 123-I MIBG imaging predicts ventricular arrhythmias in implantable cardioverter-defibrillator patients. J Am Coll Cardiol. 2010;55: 2769-2777.

25. Matsuo S, Nakamura Y, Matsumoto T, Nakae I, Murata K, Horie M. Prognostic value of iodine-123 metaiodobenzylguanidine imaging in patients with heart failure. Exp Clin Cardiol. 2003;8:95-98.

26. Zhao C, Shuke N, Yamamoto W, et al. Comparison of cardiac sympathic nervous function with left ventricular function and perfusion in cardiomyopathies by ${ }^{123} \mathrm{I}-$ MIBG SPECT and ${ }^{99 \mathrm{~m}}$ Tc-tetrofosmin electrocardiographically gated SPECT. $J$ Nucl Med. 2001;42:1017-1024.

27. Bengel FM, Barthel P, Matsunari I, Schmidt G, Schwaiger M. Kinetics of ${ }^{123}$ IMIBG after acute myocardial infarction and reperfusion therapy. J Nucl Med. 1999;40:904-910.

28. Verberne HJ, Habraken JBA, van Eck-Smit BLF, Agostini D, Jacobson AF. Variations in ${ }^{123}$ I-metaiodobenzylguanidine (MIBG) late heart mediastinal ratios in chronic heart failure: a need for standardisation and validation. Eur J Nucl Med Mol Imaging. 2008;35:547-553.

29. Matsuo S, Takahashi M, Nakamura Y, Kinoshita M. Evaluation of cardiac sympathetic innervation with iodine-123-metaiodobenzylguanidine imaging in patients with silent myocardial ischemia. J Nucl Med. 1996;37:712-717.

30. Scott LA, Kench PL. Cardiac autonomic neuropathy in the diabetic patient: does ${ }^{123}$ I-MIBG imaging have a role to play in early diagnosis? J Nucl Med Technol. 2004;32:66-71. 